\title{
Search for low-mass PMS companions around X-ray selected late B stars ${ }^{\star}$
}

\author{
S. Hubrig ${ }^{1}$, D. Le Mignant ${ }^{2,3}$, P. North ${ }^{4}$, and J. Krautter ${ }^{5}$ \\ 1 European Southern Observatory, Casilla 19001, Santiago 19, Chile \\ 2 Laboratoire d'Astrophysique, Observatoire de Grenoble, BP 53, 38041 Grenoble, France \\ 3 W. M. Keck Observatory, 65-1120 Mamalahoa Highway, Kamuela, HI 96743, USA \\ 4 Institut d'Astronomie de l'Université de Lausanne, 1290 Chavannes-des-Bois, Switzerland \\ 5 Landessternwarte Heidelberg-Königstuhl, 69117 Heidelberg, Germany
}

Received 20 September 2000 / Accepted 15 March 2001

\begin{abstract}
We have observed 49 X-ray-detected bright late B-type dwarfs to search for close low-mass pre-main sequence (PMS) companions using the European Southern Observatory's ADONIS (Adaptive Optics Near Infrared System) instrument. We announce the discovery of 21 new companions in 9 binaries, 5 triple, 4 quadruple system and 1 system consisting of five stars. The detected new companions have $K$ magnitudes between 6.5 and $17 .{ }^{\mathrm{m}} 3$ and angular separations ranging from $0 .{ }^{\prime \prime} 2$ to 14 .' $^{\prime}$ (18-2358 AU).
\end{abstract}

Key words. stars: binaries: visual - stars: early type - stars: pre-main-sequence - X-rays: stars

\section{Introduction}

In the ROSAT All-sky Survey (RASS), high X-ray fluxes, up to $10^{31} \mathrm{erg} / \mathrm{s}$, were found for 86 late B-type dwarfs of spectral types B7-B9 (Berghöfer et al. 1996). This was very surprising, since from a theoretical point of view stars in the spectral range B5-A7 should not have any significant X-ray emission. This was essentially confirmed for B-type stars by Grillo et al. (1992) from their Einstein Observatory SAO-based catalog of B-type stars. In the few cases when X-ray emission had been detected in such B5-A7 stars, it was found to originate from a cool companion. In order to search for the source of the X-rays, $17 \mathrm{~B}$ stars of the RASS sample which were known to be visual binaries, were observed with ROSAT's HighResolution Imager (HRI), which has a spatial resolution of 5 arcsec. Quite unexpectedly, only in a few cases could the X-ray emission be linked to the known visual companion (Berghöfer \& Schmitt 1994; Berghöfer \& Schmitt 2001). Berghöfer et al. (1997) noticed that $77 \%$ of the RASS detected stars are known spectroscopic binaries. It is striking that, for a large number of these systems, the observed X-ray fluxes are much too high to be explained by main sequence SB secondaries of spectral type $\mathrm{F}, \mathrm{G}$, $\mathrm{K}$ and $\mathrm{M}$ having $L_{\mathrm{X}} \leq 10^{29} \mathrm{ergs}^{-1}$ (Schmitt 1997). The most likely source of the X-rays might, therefore, be a very

Send offprint requests to: S. Hubrig, e-mail: Shubrig@eso.org

* Based on observations obtained at the European Southern Observatory, La Silla, Chile (ESO programme No. 62.I-0477, and Swiss $70 \mathrm{~cm}$ photometric telescope). active late-type companion, such as a PMS star or a very young star on the main sequence. This hypothesis is also supported by the fact that a significant fraction of the late-B stars found in the ROSAT survey belongs to rather young stellar groups: Sco-Cen association, Sco OB2, and the Pleiades cluster and supercluster.

It was shown that approximately two-thirds of all solar-type field stars are members of multiple systems (e.g. Duquennoy \& Mayor 1991), and the fraction of stars which are formed in binary or multiple systems may be even higher (e.g. Ghez et al. 1993; Leinert et al. 1993). However, only a few systems with highly differing masses are known. A pioneering study had been carried out by Gahm et al. (1983) and by Lindroos (1986) who found 78 likely pairs with an early-type and a late-type companion. X-ray observations from a part of the Lindroos systems were carried out by Huelamo et al. (2000); they found several of the late-type companions to be bona fide PMS stars.

The scientific interest in binarity studies of higher mass stars derives from the question of whether the different processes of high-mass and low-mass star formation (Shu et al. 1987) reflect themselves in different binary frequencies and distributions of mass ratios.

One method to study whether late B-type stars are intrinsic X-ray emitters or whether the detected X-ray emission originates from a hitherto undetected companion is diffraction-limited imaging in the optical or infrared spectral range on a sample of X-ray-selected late B-type stars. 
Optical speckle methods are not suitable for the detection of possible X-ray emitting PMS companions, as the magnitude differences between the bright early-type primaries and the faint late-type secondaries in the visual is rather extreme, i.e. of the order of 8-10 magnitudes. However, in the near-infrared $(J, H, K)$ the contrast between the primary and secondary components is much more favorable, i.e. only 4-5 magnitudes difference. This much improved contrast in the infrared and the fact that the primaries are very bright visual objects $(V=1.8-6.5)$ made this a perfect project for high angular resolution imaging using a high order adaptive optics system.

In Sect. 2, we describe the observations performed with the ADONIS instrument and the data reduction procedure. In Sect. 3, we present the results. In Sect. 4, we discuss the implication of these results for the formation and evolution of binary and multiple stars.

\section{Observations and data reduction}

A total of 49 late-type X-ray emitting B dwarfs were observed. The observations were performed with the ESO adaptive optics system ADONIS and SHARPII+ (System for High Angular Resolution Pictures) near infrared (NIR) imaging camera with a NICMOS III $256 \times$ 256 pixel array at the $3.6-\mathrm{m}$ telescope with a plate scale of $0.050 \mathrm{arcsec} / \mathrm{pixel}$, yielding a camera field-of-view of $12^{\prime \prime} .8 \times 12^{\prime \prime} .8$. Since all observed objects are bright stars they were used as reference stars for wavefront sensing. Forty stars were observed in the normal imaging mode in the $K$ band during two nights in March 1999. Whenever the star appeared to be multiple, it was immediately observed in the $J$ and $H$ bands. The $K$-band survey was the priority during these nights. A few companions could be detected from $K$ band images only during the later careful data reduction. For those objects, only $K$-band images are available.

The AO system and NIR camera settings time overheads are of the order of a few minutes for every target in such a survey. Given the time constraints, we decided: 1) not to optimize the $\mathrm{AO}$ system performance when switching to the next target and the result of such a choice was the lower resolution in $J$ and $H$ bands; 2 ) to spend the minimum time to adjust the individual integration time, although the PSF peak intensity may vary by more than $10 \%$ between consecutive frames. Therefore, for a few systems, the central pixels in the image core of the primary star are saturated. As a result, the photometric calibration for these stars provides only a lower limit of the $K$ magnitude.

Every target was observed at five positions on the NIR array using a dither pattern. As a result, the size for the observed area is of $24^{\prime \prime} \times 24^{\prime \prime}$ centered on the target. For every position, we recorded 16 frames with an individual integration time between 300 and $1200 \mathrm{~ms}$, depending on the star brightness. For the stars with detected companions, we have subsequently observed calibrator stars for point spread function (PSF) measurements and
Table 1. The list of X-ray selected late B-type stars.

\begin{tabular}{|c|c|c|c|c|}
\hline HD & $V$ & Sp. type & $\log L_{\mathrm{X}}{ }^{1}$ & Remarks \\
\hline 1685 & 5.50 & B9V & 29.89 & $\mathrm{CM}^{\mathrm{a}}$ \\
\hline 21790 & 4.73 & B9V & 29.47 & $\mathrm{CM}$ \\
\hline 27376 & 3.55 & B9Vmnp & 28.54 & $\mathrm{CM}, \mathrm{SB} 2^{\mathrm{b}}+$ vis. comp. ${ }^{\mathrm{c}}$ \\
\hline 27657 & 5.87 & B9IV & 29.76 & CM, vis. comp. \\
\hline 29589 & 5.45 & B8mnp & 30.74 & $\mathrm{CM}, \mathrm{SB} 1^{\mathrm{d}}$ \\
\hline 32964 & 5.10 & B9mnp & 29.80 & $\mathrm{SB} 2+$ vis. comp. \\
\hline 33802 & 4.46 & $\mathrm{~B} 8 \mathrm{~V}$ & 30.75 & vis. comp. \\
\hline 33904 & 3.28 & B9mnp & 30.02 & $\mathrm{CM}$ \\
\hline 36408 & 5.46 & B7III & 29.64 & SB1 + vis. comp. \\
\hline 39780 & 6.18 & B9.5IV & 29.44 & $\mathrm{CM}, \mathrm{SB} 2+$ vis. comp. \\
\hline 40964 & 6.59 & $\mathrm{~B} 8 \mathrm{~V}$ & 30.23 & vis. comp. \\
\hline 49333 & 6.08 & B7Hew & 30.31 & \\
\hline 50860 & 6.47 & $\mathrm{~B} 8 \mathrm{~V}$ & 30.71 & vis. comp. \\
\hline 73340 & 5.78 & B8p & 30.38 & \\
\hline 73952 & 6.45 & $\mathrm{~B} 8 \mathrm{~V}$ & 29.73 & vis. comp. \\
\hline 74067 & 5.20 & B9V & 29.27 & vis. comp. \\
\hline 75333 & 5.31 & B9mnp & 30.04 & \\
\hline 78556 & 5.61 & B9.5Si & 29.72 & SB1 + vis. comp. \\
\hline 79469 & 3.88 & B9.5V & 29.20 & SB1 + vis. comp. \\
\hline 83944 & 4.51 & B9IV-V & 29.29 & SB2 \\
\hline 90972 & 5.57 & B9.5V & 29.53 & SB2 + vis. comp. \\
\hline 92664 & 5.50 & B9Si & 29.78 & \\
\hline 100841 & 3.12 & B9III & 29.38 & vis. comp. \\
\hline 108767 & 2.95 & B9.5V & 28.71 & vis. comp. \\
\hline 110073 & 4.63 & B8mnp & 30.13 & SB1 \\
\hline 113902 & 5.71 & $\mathrm{~B} 8 \mathrm{~V}$ & 30.42 & \\
\hline 114911 & 4.77 & $\mathrm{~B} 8 \mathrm{~V}$ & 30.10 & SB2 + vis. comp. \\
\hline 118354 & 5.89 & $\mathrm{~B} 8 \mathrm{~V}$ & 30.10 & \\
\hline 119055 & 5.75 & AIV & 29.94 & vis. comp. \\
\hline 126981 & 5.50 & $\mathrm{~B} 8 \mathrm{~V}$ & 30.07 & \\
\hline 129956 & 5.67 & B9.5V & 29.28 & \\
\hline 133880 & 5.76 & B8IV & 30.29 & \\
\hline 134837 & 6.08 & $\mathrm{~B} 8 \mathrm{~V}$ & 29.70 & \\
\hline 134946 & 6.47 & B8III & 30.00 & vis. comp. \\
\hline 135734 & 4.27 & $\mathrm{~B} 8 \mathrm{~V}$ & 29.97 & vis. comp. \\
\hline 141556 & 3.95 & B9hgmn & 29.79 & SB2 \\
\hline 144334 & 5.92 & B9Si & 30.19 & \\
\hline 145483 & 5.67 & B9V & 30.41 & vis. comp. \\
\hline 158094 & 3.60 & $\mathrm{~B} 8 \mathrm{~V}$ & 29.04 & vis. comp. \\
\hline 159376 & 6.48 & B9Si & 30.14 & \\
\hline 169022 & 1.80 & B9.5III & 29.99 & vis. comp. \\
\hline 169978 & 4.61 & B7.5III & 29.99 & \\
\hline 176270 & 6.40 & B8IV-V & 30.43 & SB1 + vis. comp. \\
\hline 177756 & 3.43 & B9V & 28.47 & \\
\hline 180555 & 5.63 & B9.5 & 29.45 & vis. comp. \\
\hline 181869 & 3.95 & $\mathrm{~B} 8 \mathrm{~V}$ & 29.57 & SB1 \\
\hline 184707 & 4.60 & B8/B9V & 30.40 & vis. comp. \\
\hline 221507 & 4.37 & B9.5mnp & 29.58 & $\mathrm{CM}$ \\
\hline 222847 & 5.24 & B9V & 29.78 & $\mathrm{CM}$ \\
\hline
\end{tabular}

${ }^{1} \log L_{\mathrm{X}}$ values are taken from Berghöfer et al. (1996).

a CM: coronographic mode.

b SB2: double-lined spectroscopic binary.

c vis. comp.: the visual companion already known from the literature.

d SB1: spectroscopic binary known from the literature. 
a posteriori deconvolution. Photometric standards were observed under the same conditions to get absolute JHK photometry. As measured by the La Silla Differential Image Motion Monitor (DIMM), the atmospheric seeing during our run varied with time, ranging from $0 . \prime 8$ to $1^{\prime \prime} .2$. In the $K$-band, the diffraction limit was reached at all times $(F W H M \approx 0 \prime \prime 13)$, with the Strehl ratio ranging from 15 to $35 \%$. In the $J$ and $H$ bands the images are not diffraction-limited with the angular resolution varying from 0 !' 13 to 0 "' 20 . Additionally, 9 late-type X-ray emitting B dwarf stars were imaged with the coronographic mask during the night of 26 September 1999. Thus, the whole sample of studied stars consists of 49 objects.

In Table 1 we present the list of the observed late Btype stars. Their visual magnitudes and spectral types were retrieved from the SIMBAD data base. The X-ray luminosity $\log L_{\mathrm{X}}$ given in Col. 4 was taken from Berghöfer et al. (1996). In the last column we give some remarks about the observing mode and binarity.

For the data reduction we used our own C-shell scripts based on the ESO/eclipse data reduction $\mathrm{C}$ routines (Devillard 1997). The background emission was computed for every pixel as a median value of the stack of $16 \times 5$ frames. For each exposure we subtracted background sky emission, divided by flat field, corrected for bad pixels and used shift-and-add method to yield the final images.

The photometry was performed using the IRAF/DIGIPHOT package. For the systems with an angular separation larger than $2^{\prime \prime}$ we applied a standard aperture photometry method. For other systems we combined PSF fitting of the components and aperture photometry to get the absolute fluxes of the stars.

For a few systems with angular separation smaller than 0.' 4, we cross-checked PSF fitting with PLucy deconvolution, which is provided by IRAF Space Telescope Science Data Analysis System. No discrepancy was found between PSF fitting and PLucy deconvolution results, either for photometric fluxes or for star astrometric positions. The average rms error is $0.03 \mathrm{mag}$ for differential photometry, $0.05 \mathrm{mag}$ for absolute photometry, $0 . \prime 005$ on the projected separation, and $0.2^{\circ}$ on the position angle. For objects observed in the coronographic mode, the average rms error for absolute photometry is $0.1 \mathrm{mag}, 0$.'05 for the projected separation and $0.2^{\circ}$ for the position angle, respectively.

The detection limits for these observations depend on the separation range, as we cannot detect any companions below $0.1^{\prime \prime}$, and on the contrast between primary and companion(s). This contrast also depends on separation: photon noise prevents from detecting very close binaries (0.1 to $\left.0.3^{\prime \prime}\right)$, while detector read-out noise is the limiting factor for wider systems. The detection limit values estimated for our observational data set are: $\Delta K \sim 2$ at separations of $\sim 0.3^{\prime \prime}$ and below, $\Delta K \sim 5$ at separations $\sim 2^{\prime \prime}$ and $\Delta K \sim 9$ at separations of $\sim 4^{\prime \prime}$ and beyond.

Most stars had been measured in the GENEVA photometric system (Golay 1980; Rufener \& Nicolet 1988; Cramer 1999), with the photoelectric photometer P7 (Burnet \& Rufener 1979) installed on the $70 \mathrm{~cm}$ Swiss telescope at La Silla (ESO, Chile). The photometric data in the GENEVA system are collected in the General Catalogue (Rufener 1988) and its up-to-date database (Burki et al. 2001).

\section{Results}

For 25 of our 49 X-ray selected late B-type stars we could find companions in the scanned $24^{\prime \prime} \times 24^{\prime \prime}$ area. Six of these systems were already known to be visual binaries. The discovered companions have $K$ magnitudes between 6.5 and $17^{\mathrm{m}} 3$ and angular separations ranging from 0.2 to $14^{\prime \prime} .1$.

The photometric and astrometric results of our observations are presented in Table 2, where we list the HD numbers; the separations and position angles; $J, H$ and $K$ magnitudes; the magnitude difference $\Delta K$ between the primary and the companion in the $K$-band; and the $J-H$ and $H-K$ colors. Only in two systems - HD 1685 and HD 73952 - do the companions have a considerable $(\Delta(H-K) \geq 0.2 \mathrm{mag}) \mathrm{NIR}$ excess. Usually, NIR excess betrays the existence of substantial amounts of circumstellar matter around the stars.

\subsection{Probability for random pairing}

In order to check whether some of our late-type binary identifications could be due to a random positional coincidence with field stars, we calculated the probability $P$ that a stellar $K$-band source occurs by random coincidence in the total area of our image fields at given $K$ magnitude.

For our calculations, we used the density of infrared $K$-band sources as measured by both DENIS (Deep NearInfrared Survey of the Southern Sky; Epchtein 1998) and 2MASS surveys (Two Microns All Sky Survey ${ }^{1}$; Skrutskie 2000). For DENIS, it was not possible to calculate individual numbers for the different galactic latitudes of our target stars, since star counts from this survey have so far been published only for a few selected fields. In order to get conservative upper limits, we used the field with the highest $K$-band star count density we found in the literature. This is a one-square-degree field centered around the galactic coordinates $l=331^{\circ}, b=-1.81^{\circ}$ (Ruphy 1998). This field is in a very crowded area close to the galactic plane.

The probabilities for the presence of a background star for our total ADONIS image field of view of $24^{\prime \prime} \times 24^{\prime \prime}$ are presented in Table 3 for a number of $K$-band magnitudes.

For 2MASS, we could compute the probability of finding a companion for 14 out of the 19 systems found, by counting stars of a given magnitude in a circle centered on the primary and with a $1^{\circ}$ radius. Sorting the companions by their $K$ magnitudes and selecting the largest probability in each 0.5 magnitude bin, we obtain the numbers listed in Col. 3 of Table 3. The very large probability

\footnotetext{
1 see http://irsa.ipac.caltech.edu/applications/Stats
} 
Table 2. Photometry and astrometry of X-ray selected late B-type stars with companions.

\begin{tabular}{|c|c|c|c|c|c|c|c|c|c|}
\hline \multicolumn{10}{|c|}{ Detected visual systems } \\
\hline $\mathrm{HD}$ & Comp. & $\rho\left({ }^{\prime \prime}\right)$ & $\mathrm{PA}\left({ }^{\circ}\right)$ & $J$ & $H$ & $K$ & $\Delta K$ & $J-H$ & $H-K$ \\
\hline HD 1685 & $\begin{array}{l}\mathrm{A} \\
\mathrm{B}\end{array}$ & 2.28 & 211.4 & 11.7 & 10.3 & 10.1 & & 1.4 & 0.2 \\
\hline HD 21790 & $\begin{array}{l}\mathrm{A} \\
\mathrm{B}\end{array}$ & 14.12 & 114.5 & & & 16.2 & & & \\
\hline HD 27376 & $\begin{array}{l}\mathrm{A} \\
\mathrm{B}\end{array}$ & 5.32 & 162.5 & 10.7 & 10.0 & 9.9 & & 0.7 & 0.1 \\
\hline HD 29589 & $\begin{array}{l}\mathrm{A} \\
\mathrm{B}\end{array}$ & 10.00 & 251.3 & & & 17.3 & & & \\
\hline HD 32964 & $\begin{array}{l}\mathrm{A} \\
\mathrm{B}\end{array}$ & 1.613 & 232.6 & $\begin{array}{l}\leq 5.19 \\
10.03\end{array}$ & $\begin{array}{c}\leq 5.22 \\
9.37\end{array}$ & $\begin{array}{c}\leq 5.22 \\
9.38 \\
\end{array}$ & $\geq 4.16$ & 0.66 & 0.01 \\
\hline HD 33802 & $\begin{array}{l}\mathrm{A} \\
\mathrm{B}\end{array}$ & 0.371 & 22.9 & $\begin{array}{l}4.73 \\
6.92\end{array}$ & $\begin{array}{l}4.78 \\
6.90\end{array}$ & $\begin{array}{l}4.78 \\
7.22\end{array}$ & 2.44 & $\begin{array}{c}-0.05 \\
0.02\end{array}$ & $\begin{array}{c}0.0 \\
-0.32\end{array}$ \\
\hline HD 73340 & $\begin{array}{l}\mathrm{A} \\
\mathrm{B}\end{array}$ & 0.604 & 221.2 & $\begin{array}{l}5.97 \\
9.32\end{array}$ & $\begin{array}{l}6.10 \\
8.71\end{array}$ & $\begin{array}{l}6.13 \\
8.65\end{array}$ & 2.52 & $\begin{array}{c}-0.13 \\
0.61\end{array}$ & $\begin{array}{c}-0.03 \\
0.06\end{array}$ \\
\hline HD 73952 & $\begin{array}{l}\mathrm{A} \\
\mathrm{B}\end{array}$ & 1.162 & 205.3 & $\begin{array}{l}6.62 \\
11.8\end{array}$ & $\begin{array}{c}6.57 \\
11.25\end{array}$ & $\begin{array}{c}6.63 \\
10.82\end{array}$ & 4.19 & $\begin{array}{l}0.05 \\
0.55\end{array}$ & $\begin{array}{c}-0.06 \\
0.43\end{array}$ \\
\hline HD 75333 & $\begin{array}{l}\mathrm{A} \\
\mathrm{B}\end{array}$ & 1.340 & 165.8 & $\begin{array}{c}5.40 \\
10.21\end{array}$ & $\begin{array}{l}5.45 \\
9.42\end{array}$ & $\begin{array}{l}5.49 \\
9.45\end{array}$ & 3.96 & $\begin{array}{c}-0.05 \\
0.79\end{array}$ & $\begin{array}{l}-0.04 \\
-0.03\end{array}$ \\
\hline HD 110073 & $\begin{array}{l}\mathrm{A} \\
\mathrm{B}\end{array}$ & 1.192 & 75.0 & $\begin{array}{l}4.74 \\
8.29\end{array}$ & $\begin{array}{l}4.78 \\
8.00\end{array}$ & $\begin{array}{l}4.85 \\
7.93\end{array}$ & 3.08 & $\begin{array}{c}-0.04 \\
0.29\end{array}$ & $\begin{array}{c}-0.07 \\
0.07\end{array}$ \\
\hline HD 114911 & $\begin{array}{l}\mathrm{A} \\
\mathrm{B}\end{array}$ & 2.706 & 124.6 & $\begin{array}{c}5.06 \\
10.31\end{array}$ & $\begin{array}{l}5.07 \\
9.61\end{array}$ & $\begin{array}{l}5.11 \\
9.43\end{array}$ & 0.319 & $\begin{array}{c}-0.01 \\
0.70\end{array}$ & $\begin{array}{c}-0.04 \\
0.18\end{array}$ \\
\hline HD 133880 & $\begin{array}{l}\mathrm{A} \\
\mathrm{B}\end{array}$ & 1.222 & 109.2 & $\begin{array}{l}5.94 \\
9.01\end{array}$ & $\begin{array}{l}5.99 \\
8.55\end{array}$ & $\begin{array}{l}6.06 \\
8.41\end{array}$ & 2.35 & $\begin{array}{c}-0.05 \\
0.46\end{array}$ & $\begin{array}{c}-0.07 \\
0.14\end{array}$ \\
\hline HD 134837 & $\begin{array}{l}\mathrm{A} \\
\mathrm{B}\end{array}$ & 4.696 & 154.3 & & & $\begin{array}{l}\leq 6.33 \\
10.99\end{array}$ & $\geq 4.66$ & & \\
\hline HD 134946 & $\begin{array}{l}\mathrm{A} \\
\mathrm{B}\end{array}$ & 8.212 & 45.3 & & & $\begin{array}{l}\leq 6.47 \\
12.51\end{array}$ & $\geq 6.04$ & & \\
\hline HD 135734 & $\begin{array}{l}\mathrm{A} \\
\mathrm{B} \\
\mathrm{C}\end{array}$ & $\begin{array}{l}1.038 \\
6.146\end{array}$ & $\begin{array}{l}322.5 \\
156.9\end{array}$ & & & $\begin{array}{l}5.11 \\
5.32 \\
14.7\end{array}$ & $\begin{array}{l}0.21 \\
9.59\end{array}$ & & \\
\hline HD 145483 & $\begin{array}{l}\mathrm{A} \\
\mathrm{B} \\
\mathrm{C}\end{array}$ & $\begin{array}{l}3.767 \\
0.202\end{array}$ & $\begin{array}{c}70.8 \\
39.16\end{array}$ & & & $\begin{array}{c}\leq 5.94 \\
6.85 \\
7.94\end{array}$ & $\begin{array}{l}\geq 0.91 \\
\geq 2.00\end{array}$ & & \\
\hline HD 159376 & $\begin{array}{l}A \\
B \\
C \\
D\end{array}$ & $\begin{array}{l}8.703 \\
5.420 \\
6.340\end{array}$ & $\begin{array}{c}52.9 \\
10.4 \\
141.6\end{array}$ & & & $\begin{array}{c}\leq 6.32 \\
12.66 \\
14.18 \\
15.07\end{array}$ & $\begin{array}{l}\geq 6.34 \\
\geq 7.86 \\
\geq 8.75\end{array}$ & & \\
\hline HD 169022 & $\begin{array}{l}\mathrm{A} \\
\mathrm{B}\end{array}$ & 2.392 & 142.3 & $\begin{array}{l}1.79 \\
6.67\end{array}$ & $\begin{array}{l}1.80 \\
6.45\end{array}$ & $\begin{array}{l}1.82 \\
6.50\end{array}$ & 4.68 & $\begin{array}{c}-0.01 \\
0.22\end{array}$ & $\begin{array}{l}-0.02 \\
-0.05\end{array}$ \\
\hline HD 169978 & $\begin{array}{l}\mathrm{A} \\
\mathrm{B}\end{array}$ & 3.085 & 168.7 & & & $\begin{array}{l}\leq 4.92 \\
13.69\end{array}$ & $\geq 8.77$ & & \\
\hline & Star & already & nown to & mem & rs of vi & dal bina & y syster & & \\
\hline HD 27657 & $\begin{array}{l}\mathrm{A} \\
\mathrm{B}\end{array}$ & 4.131 & 2.8 & & & $\begin{array}{c}7.0 \\
8.15\end{array}$ & 1.15 & & \\
\hline HD 36408 & $\begin{array}{l}A \\
B\end{array}$ & 9.742 & 141.0 & & & $\begin{array}{l}6.09 \\
6.34\end{array}$ & 0.25 & & \\
\hline HD 78556 & $\begin{array}{l}\mathrm{A} \\
\mathrm{B}\end{array}$ & 1.300 & 298.5 & & & $\begin{array}{l}5.65 \\
8.96\end{array}$ & 3.31 & & \\
\hline HD 90972 & $\begin{array}{l}\mathrm{A} \\
\mathrm{B}\end{array}$ & 11.084 & 225.8 & & & $\begin{array}{l}5.63 \\
8.34\end{array}$ & 2.71 & & \\
\hline HD 119055 & $\begin{array}{l}\mathrm{A} \\
\mathrm{B}\end{array}$ & 4.688 & 134.2 & & & $\begin{array}{c}\leq 5.73 \\
8.21\end{array}$ & $\geq 2.48$ & & \\
\hline HD 184707 & $\begin{array}{l}\mathrm{A} \\
\mathrm{B}\end{array}$ & 2.435 & 173.1 & & & $\begin{array}{c}\leq 4.83 \\
7.59\end{array}$ & $\geq 2.76$ & & \\
\hline
\end{tabular}


Table 3. Upper limits for probabilities $P$ of presence of background sources for various $K$-band magnitudes.

\begin{tabular}{rll}
\hline$K[\mathrm{mag}]$ & $P($ DENIS $)$ & $P_{\max }(2 \mathrm{MASS})$ \\
\hline 6 & 0.0004 & 0.0007 \\
7 & 0.002 & 0.0002 \\
8 & 0.006 & 0.0007 \\
9 & 0.012 & 0.001 \\
10 & 0.028 & 0.001 \\
11 & 0.067 & 0.009 \\
12 & 0.116 & - \\
13 & 0.200 & 0.93 \\
\hline
\end{tabular}

given for $K=13$ comes from HD 159376, which lies in an exceptionally crowded field.

The DENIS numbers are very conservative upper limits only, since they were calculated on the basis of the most pessimistic assumptions. First, all of our objects are located at higher galactic latitudes, where the star counts drastically decrease. For instance, as shown by Ruphy et al. (1997) for areas located at $l=303^{\circ}$, the star counts decrease by about a factor of 10 between $b=2.37^{\circ}$ and $b=-14.63^{\circ}$. Second, they were calculated for the whole field of $576 \operatorname{arcsec}^{2}$, whereas the mean distance of the companion stars found is 4.3 arcsec only.

It is worth noting that out of 29 detected companions in our $24^{\prime \prime} \times 24^{\prime \prime}$ field $K$ images, 17 are found at a distance less than $5^{\prime \prime}$ from the primary (note that 8 out of the 29 companions detected were already known). 27 of the 29 detected companions are at distances smaller than or equal to $10^{\prime \prime}$. In other words, $93 \%$ of the detected companions are found in the central $55 \%$ of the scanned area around the primary. Our estimate is that on average the probabilities for a random positional coincidence of our sources are at least a factor of two lower than the numbers in Table 3. In view of these facts, we can safely assume that - with the exception of the six sources fainter than 13th magnitude - random positional coincidences do not play a significant role. For HD 21790 and HD 29589 where objects with $K=16.2$ and $K=17.3$ were found at relatively large distances of $14^{\prime \prime} 12$ and $10^{\prime \prime}$ arcsec, respectively, a random positional coincidence can definitely not be excluded. These conclusions are entirely confirmed by the 2MASS results, which add much weight to them.

\subsection{Fundamental parameters of primaries}

In Table 4 we present the basic data for the late-B primaries. We give the distances $d$ calculated from Hipparcos parallaxes, relative uncertainties of parallaxes, absolute visual magnitudes, bolometric luminosities, effective temperatures, masses (in solar masses) and the ages. We only accepted those parallaxes which exceed their corresponding error by at last a factor of three. This criterion is not fulfilled for one star only in our sample, HD 36408, which was already known to have a visual companion at the distance $99^{\prime \prime} 74$ before our observations. In the last column we give values for $L_{\mathrm{X}}$. We use the standard equations (see e.g. Berghöfer et al. 1996, Eqs. (2) through (5)) to calculate $L_{\mathrm{X}}, M_{V}$ and $L_{\mathrm{Bol}}$. For the calculation of $L_{\mathrm{X}}$ we use Hipparcos values for the distances and $f_{\mathrm{X}}$ values from Berghöfer et al. (1996). We assume $R=3.1$ for the standard interstellar reddening law in which the $E(B-V)$ values have been calculated from $(B-V)$ colors taken from the Bright Star Catalogue (1982, henceforth BSC) and from intrinsic $(B-V)_{0}$ colors for late B-type stars taken from FitzGerald (1970). Finally, the bolometric luminosities were calculated using bolometric corrections published by Schmidt-Kaler (1982).

The effective temperatures of all stars but one (HD 119055) were determined using Geneva photometry calibrated by Künzli et al. (1997), assuming a metallicity $[\mathrm{M} / \mathrm{H}]=0.0$. The most appropriate grid was chosen, i.e. that of the reddening-free $X$ and $Y$ parameters, which are mostly sensitive to $T_{\text {eff }}$ and luminosity respectively (Cramer \& Maeder 1979). The formal errors given by the code are propagated from typical photometric errors and are generally lower than $100 \mathrm{~K}$. They are optimistic in the sense that they are only random errors and do not include possible systematic errors linked to the calibration or to cosmic causes such as unresolved duplicity. In the case of the Bp Si stars HD 78556, HD 133880 and HD 159376, the temperature was determined using the recipe given by North (1998), still using Geneva photometry. For them, the error on $T_{\text {eff }}$ was arbitrarily set to $300 \mathrm{~K}$ because the temperature estimate is less reliable than for normal stars.

The effective temperature of HD 119055 was determined by interpolating in the uvby $\beta$ grid of Strömgren indices computed for $[\mathrm{M} / \mathrm{H}]=0.0$, from ATLAS9 models and fluxes (Castelli 2000). Observed Strömgren indices were taken from the catalogue of Mermilliod et al. (1997) and were dereddened using the UVBYLIST code of Moon (1985). The errors associated with the parameters were derived by assuming an uncertainty of $\pm 0.015 \mathrm{mag}$ for all the observed indices, except $\beta$, for which a probable error of $\pm 0.005 \mathrm{mag}$ was adopted. The accuracy of the determination of the effective temperatures based on Strömgren photometry is better than $100 \mathrm{~K}$.

For the star HD 36408, which has a very bad parallax accuracy, all fundamental parameters are based on purely photometric estimates.

To derive the masses and ages of late B-type primaries, we used the grids of stellar models provided by Schaller et al. (1992). The luminosities were estimated from the Hipparcos parallaxes (except for HD 36408, for which they were obtained from photometric $T_{\text {eff }}, \log g$ and from evolutionary tracks) and from visual absorption obtained from Geneva $X$ and $Y$ photometric parameters through the calibration of Cramer (1982), assuming $A_{v} / E[B-V]=3.65$. The bolometric corrections are those of Schmidt-Kaler (1982).

The masses were interpolated in the evolutionary tracks (using a code by PN) while the ages were interpolated in the isochrones of the same models (using a code kindly made available by Drs. C. Jordi and F. Figueras). 
Table 4. Fundamental parameters of late-B primaries with good Hipparcos parallaxes.

\begin{tabular}{|c|c|c|c|c|c|c|c|c|}
\hline \multicolumn{9}{|c|}{ Detected visual systems } \\
\hline $\mathrm{HD}$ & $d(\mathrm{pc})$ & $\sigma(\pi) / \pi$ & $M_{V}$ & $\log \left(L / L_{\odot}\right)$ & $\log \left(T_{\text {eff }}\right)$ & $M / M_{\odot}$ & lo & $\log L_{\mathrm{X}}^{1}$ \\
\hline HD 1685 & 93.9 & 0.048 & 0.57 & $1.870 \pm 0.068$ & $4.016 \pm 0.006$ & $2.717 \pm 0.080$ & $8.433 \pm 0.032$ & 29.79 \\
\hline HD 21790 & 116.7 & 0.085 & -0.68 & $2.473 \pm 0.088$ & $4.064 \pm 0.002$ & $3.659 \pm 0.147$ & $8.251 \pm 0.023$ & 29.83 \\
\hline HD 27376 & 54.7 & 0.030 & -0.20 & $2.378 \pm 0.049$ & $4.106 \pm 0.002$ & $3.640 \pm 0.069$ & $8.107 \pm 0.022$ & 28.71 \\
\hline HD 29589 & 105.7 & 0.082 & 0.19 & $2.344 \pm 0.085$ & $4.161 \pm 0.003$ & $3.876 \pm 0.127$ & $7.041 \pm 1.158$ & 30.33 \\
\hline HD 32964 & 85.8 & 0.063 & 0.37 & $2.012 \pm 0.073$ & $4.045 \pm 0.002$ & $2.953 \pm 0.084$ & $8.319 \pm 0.032$ & 29.76 \\
\hline HD 33802 & 73.9 & 0.051 & 0.01 & $2.309 \pm 0.060$ & $4.117 \pm 0.002$ & $3.602 \pm 0.078$ & $7.974 \pm 0.067$ & 30.68 \\
\hline HD 73340 & 143.1 & 0.063 & -0.13 & $2.511 \pm 0.072$ & $4.178 \pm 0.003$ & $4.226 \pm 0.111$ & $7.454 \pm 0.274$ & 30.34 \\
\hline HD 73952 & 154.8 & 0.076 & 0.40 & $2.097 \pm 0.072$ & $4.087 \pm 0.002$ & $3.207 \pm 0.083$ & $8.030 \pm 0.111$ & 29.50 \\
\hline HD 75333 & 134.2 & 0.099 & -0.46 & $2.441 \pm 0.099$ & $4.088 \pm 0.002$ & $3.670 \pm 0.155$ & $8.192 \pm 0.008$ & 30.29 \\
\hline HD 110073 & 108.8 & 92 & -0.71 & $2.585 \pm$ & $4.111 \pm 0.002$ & $3.975 \pm 0.151$ & .010 & 29.57 \\
\hline HD 114911 & 124.4 & 0.073 & -0.81 & $2.622 \pm 0.075$ & $4.106 \pm 0.002$ & $4.026 \pm 0.136$ & $8.118 \pm 0.012$ & 29.95 \\
\hline HD 133880 & 126.6 & 0.108 & 0.20 & $2.195 \pm 0.134$ & $4.079 \pm 0.011$ & $3.290 \pm 0.190$ & $8.192 \pm 0.071$ & 29.94 \\
\hline HD 134837 & 111.1 & 92 & 0.74 & $1.974 \pm 0.084$ & $4.097 \pm 0.002$ & $3.111 \pm 0.101$ & $7.022 \pm 2.126$ & 29.35 \\
\hline HD 134946 & 126.4 & 0.119 & 0.55 & $2.194 \pm 0.108$ & $4.139 \pm 0.002$ & $3.564 \pm 0.147$ & $6.152 \pm 4.000$ & 29.79 \\
\hline HD 135734 & 89.1 & 0.086 & -0.57 & $2.517 \pm 0.086$ & $4.105 \pm 0.002$ & $3.843 \pm 0.139$ & $8.134 \pm 0.008$ & 30.12 \\
\hline HD 145483 & 91.4 & 0.083 & 0.56 & $1.960 \pm 0.084$ & $4.057 \pm 0.003$ & $2.939 \pm 0.088$ & $8.177 \pm 0.103$ & 30.30 \\
\hline HD 159376 & 271.0 & 0.217 & -1.24 & $2.732 \pm 0.210$ & $4.072 \pm 0.011$ & $4.099 \pm 0.420$ & $8.178 \pm 0.078$ & 30.49 \\
\hline HD 169022 & 44.3 & 0.045 & -1.41 & $2.560 \pm 0.070$ & $3.960 \pm 0.004$ & $3.515 \pm 0.138$ & $8.366 \pm 0.045$ & 29.34 \\
\hline HD 169978 & 146.8 & 0.110 & -1.31 & $2.819 \pm 0.102$ & $4.106 \pm 0.002$ & $4.392 \pm 0.216$ & $8.080 \pm 0.034$ & 29.98 \\
\hline \multicolumn{9}{|c|}{ tars already known to be members of visual binary systems } \\
\hline HD 27657 & 142.0 & 0.082 & -0.04 & $2.253 \pm 0.086$ & $4.078 \pm 0.002$ & $3.363 \pm 0.117$ & $8.219 \pm 0.023$ & 29.59 \\
\hline HD 36408 & 342.5 & 0.538 & -2.60 & $2.705 \pm 0.165$ & $4.087 \pm 0.002$ & $4.109 \pm 0.332$ & $8.150 \pm 0.054$ & 30.05 \\
\hline HD 78556 & 275.5 & 0.267 & -1.65 & $2.727 \pm 0.275$ & $4.000 \pm 0.013$ & $3.853 \pm 0.604$ & $8.260 \pm 0.177$ & 30.29 \\
\hline HD 90972 & 147.5 & 0.109 & -0.37 & $2.303 \pm 0.101$ & $4.046 \pm 0.003$ & $3.350 \pm 0.153$ & $8.330 \pm 0.020$ & 29.86 \\
\hline HD 119055 & 92.8 & 0.084 & 0.79 & $1.745 \pm 0.088$ & $3.994 \pm 0.004$ & $2.536 \pm 0.093$ & $8.509 \pm 0.036$ & 29.94 \\
\hline HD 184707 & 58.0 & 0.052 & 0.75 & $1.859 \pm 0.079$ & $4.045 \pm 0.006$ & $2.794 \pm 0.090$ & $8.181 \pm 0.114$ & 30.22 \\
\hline
\end{tabular}

${ }^{1} \log L_{\mathrm{X}}$ values are recomputed using Hipparcos values for the distances and $f_{\mathrm{X}}$ values from Berghöfer et al. (1996).

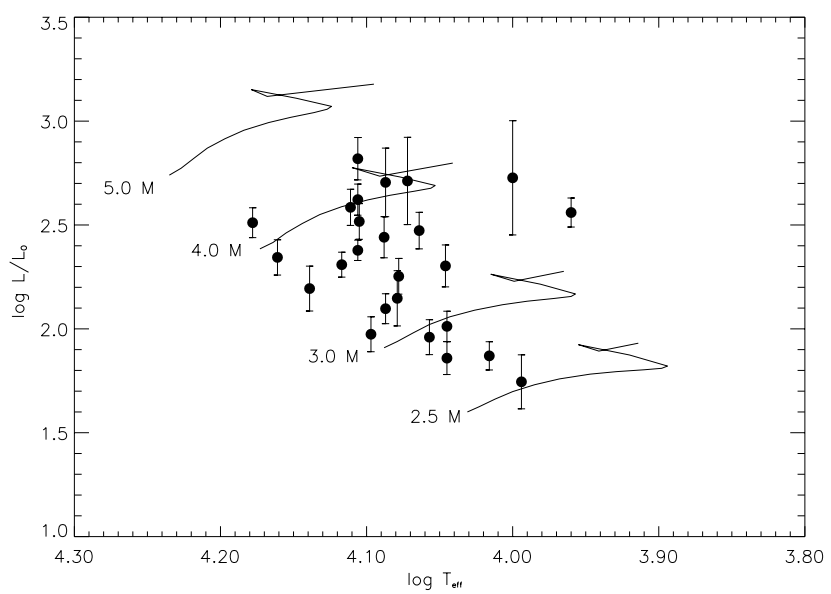

Fig. 1. Position of primaries in the $\mathrm{H}-\mathrm{R}$ diagram. The evolutionary tracks computed for stars of $2,2.5,3$, and $4 M / M_{\odot}$ by Schaller et al. (1992) are also shown.

Figure 1 shows the positions of the late B-type primaries in the $\mathrm{H}-\mathrm{R}$ diagram. For comparison we show also the evolutionary tracks of stars with masses of 2.5, 3.0, 4.0 and $5.0 M_{\odot}$.
Since the late B-type primary always dominates in the combined spectrum, we can safely assume that the measured spectral type is always that of the primary. Some uncertainty in the vertical positioning in the $\mathrm{H}-\mathrm{R}$ diagram might arise by some overestimate of the primary's luminosity due to the presence of the companion(s). In the case of a close binary consisting of two nearly identical stars, the stellar luminosity can be overestimated by $\delta \log L=0.3$. However, when only faint late-type companions are found, the actual error is negligible, as the companion luminosity is fainter by more than two orders of magnitude than the primary's. There is still a possibility that the binary nature of the star induces a measurement error for the parallax. To test Hipparcos parallaxes on multiple stars, Shatskii \& Tokovinin (1998) compared the dynamical parallaxes of visual binaries with their Hipparcos trigonometrical parallaxes. No systematic difference was found between them. The parallaxes have been found in good agreement for all distant $(\pi<15$ mas $)$ systems, while at intermediate distances $(15<\pi<30$ mas $)$ authors found cases of large errors in Hipparcos parallaxes attributable to short-period binaries that have been disregarded. Of all the systems in our sample, only three 
systems are distant systems. The closest system with a late B-type binary has a parallax $\pi=22.55$ mas.

\subsection{Fundamental parameters of companions}

In order to determine the evolutionary state of detected companions we need to know their position in the $\mathrm{H}-\mathrm{R}$ diagram or in the $M_{K}-\left(M_{J}-M_{K}\right)$ color-magnitude diagram (CMD).

Since late B-type stars are rather young, less than a few hundred million years old (Table 4), we expect that a significant fraction of the companions with low masses are PMS stars or very young main sequence stars. The masses, luminosities and effective temperatures for PMS companions can be then obtained from the location of the companions in $M_{K}-\left(M_{J}-M_{K}\right)$ color-magnitude diagram using evolutionary models for low-mass PMS stars calculated by Baraffe et al. (1998) (hereafter B98). These models include the most recent interior physics and the latest generation of non-grey atmosphere models. In fact, two sets of models of Baraffe et al. (1998), namely B98 LMS and $\mathrm{B} 98_{\mathrm{Sun}}$, can be used to derive the fundamental parameters of PMS companions. The set B98 ${ }_{\mathrm{LMS}}$ uses a helium abundance $Y=0.275$ for $[\mathrm{M} / \mathrm{H}]=0$ and a general mixing length parameter $\alpha=1 / H_{\mathrm{P}}=1.0$. It is calculated for the masses in the range from $0.020 M_{\odot}$ to $1.2 M_{\odot}$. The models B98 $8_{\text {Sun }}$ were computed to reproduce the properties of the Sun at 4.61 Gyrs, with $\alpha=1.9$ and $Y=0.282$. It is difficult to decide which mixing length parameter is the better one for PMS models. There are no theoretical arguments and the few observations available so far do not give any clear picture either. Since the models B98 ${ }_{\text {Sun }}$ are computed only for 0.7 to $1.2 M_{\odot}$ we decided to use for this work the set B98 $8_{\text {LMS }}$ with the wider mass range, although for some stars the comparison was made with the B98 sun too.

For 11 of our systems, we have observations in three bands, $J, H$ and $K$. In Figs. 2 and 3 we show the position of PMS companions in CMD for the set B98 LMS and set B98 $8_{\text {Sun }}$, respectively. The comparison of both figures reveals rather conspicuous differences in the position of the companions and, therefore, in the inferred fundamental parameters. In four systems, HD 1685, HD 32964, HD 33802 and HD 169022, the companions cannot be fitted by PMS evolutionary tracks. It is very likely that the system HD 1685 does not form a physical pair. In the systems HD 32964, HD 33802 and HD 169022, the new companions discovered are in an advanced evolutionary state, i.e. they have already evolved to the main sequence and cannot be fitted by PMS evolutionary tracks. Their position on the main sequence can be obtained by using evolutionary models for main-sequence stars calculated by Schaller et al. (1992, hereforth Sch92) or by Charbonnel et al. (1999) for masses below $0.8 M_{\odot}$. However, to apply these models to our companions we need their magnitudes in the visual bandpass, which are unknown. Therefore, we first estimated the masses of the companions in an

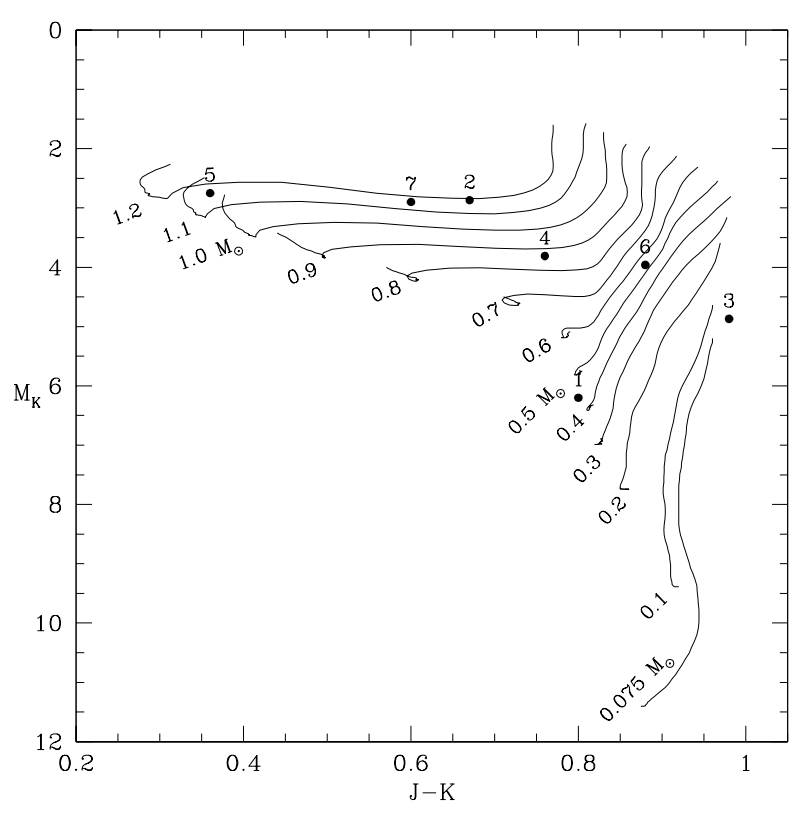

Fig. 2. Position of companions in the $M_{K}-\left(M_{J}-M_{K}\right)$ color-magnitude diagram for PMS stars using the set B98 ${ }_{\mathrm{LMS}}$. Indicated stars: HD 27376[1]; HD 73340[2]; HD 73952[3]; HD 75333[4]; HD 110073[5]; HD 114911[6]; HD 133880[7].

empirical way using $M_{(J H K)}$-mass relations (MLR) of Henry \& McCarthy (1993). These relations were determined for stars with masses 0.08 to $1.0 M_{\odot}$. Under the assumption of coeval formation of studied systems, the goal was to place in the $\mathrm{H}-\mathrm{R}$ diagram the companions with an empirical mass estimated from the $M_{(J H K)}$ MLR of Henry \& McCarthy (1993), along the isochrones of the $B$ primaries. In this way we were able to estimate their masses, luminosities and effective temperatures.

For 14 systems the only available informations are our $K$-band data. Knowing the age of late B-type primaries and assuming that systems are coeval, we placed the companions with known $M_{K}$ values along the isochrones of the $B$ primaries to estimate their masses, luminosities and effective temperatures.

Further constraints on the companion properties can be obtained from the saturation limits of $\log \left(L_{\mathrm{X}} / L_{\mathrm{bol}}\right)$ found for late-type coronal sources. They can emit up to $\approx 10^{-3}$ of their total luminosity in the soft X-ray band (Schmitt 1997). For example, a study of X-ray-discovered $\mathrm{T}$ Tauri stars in the Taurus-Auriga $\mathrm{T}$ Association (Neuhäuser et al. 1995) revealed $L_{\mathrm{X}} / L_{\mathrm{Bol}}$ values of $\log \left(L_{\mathrm{X}} / L_{\mathrm{bol}}\right)=-4.06$ for G-type and -3.56 for M-type TTS. The studies of nearby young open clusters show a considerable spread in coronal activity for stars with different rotational velocities. Although most stars appear to saturate at about $10^{-3}$ of the stellar bolometric luminosity, a few stars reach higher X-ray activity levels, up to $\log \left(L_{\mathrm{X}} / L_{\mathrm{bol}}\right) \approx-2.9 \ldots-2.8$ (Stauffer et al. 1997; Stauffer et al. 1994; Randich et al. 1995). To place a lower limit on the mass of the companion, we use the condition that the ratio $\log \left(L_{\mathrm{X}} / L_{\mathrm{bol}}\right)$ does not exceed -3 . 
Table 5. Fundamental parameters for companions using evolutionary models from Baraffe et al. (1998).

\begin{tabular}{|c|c|c|c|c|c|c|c|c|c|c|}
\hline \multicolumn{11}{|c|}{ Detected visual systems } \\
\hline $\mathrm{HD}$ & Comp. & $M_{J}$ & $M_{H}$ & $M_{K}$ & $M / M_{\odot}$ & $q$ & $\rho(\mathrm{AU})$ & $\log \left(L / L_{\odot}\right)_{B}$ & $\log \left(T_{\text {eff }}\right)_{B}$ & $\log \left(L_{\mathrm{X}} / L_{\mathrm{bol}}\right)_{B}$ \\
\hline HD 1685 & $\mathrm{~B}$ & 6.8 & 5.4 & 5.2 & 0.60 & 0.22 & 214.1 & -1.20 & 3.590 & -2.60 \\
\hline HD $21790^{\mathrm{a}}$ & $\mathrm{B}$ & & & 10.8 & 0.04 & 0.01 & 1647.8 & $<-3.83$ & 3.297 & 0.07 \\
\hline HD 27376 & $\mathrm{~B}$ & 7.0 & 6.3 & 6.2 & 0.45 & 0.12 & 291.0 & -1.46 & 3.554 & -3.42 \\
\hline HD $29589^{\mathrm{a}, \mathrm{b}}$ & B & & & 12.2 & & & 1057.0 & & & \\
\hline HD $32964^{\mathrm{c}}$ & $\mathrm{B}$ & 5.36 & 4.70 & 4.71 & 0.67 & 0.23 & 52.6 & & & \\
\hline HD 33802 & $\mathrm{~B}$ & 2.58 & 2.56 & 2.88 & & & 27.4 & & & \\
\hline HD 73340 & $\mathrm{~B}$ & 3.54 & 2.93 & 2.87 & 1.20 & 0.36 & 86.4 & -0.15 & 3.647 & -3.09 \\
\hline HD 73952 & B & 5.85 & 5.30 & 4.87 & 0.62 & 0.19 & 179.9 & -1.06 & 3.609 & -3.02 \\
\hline HD 75333 & $\mathrm{~B}$ & 4.57 & 3.78 & 3.81 & 0.88 & 0.24 & 179.8 & -0.30 & 3.695 & -3.00 \\
\hline HD 110073 & $\mathrm{~B}$ & 3.11 & 2.82 & 2.75 & 1.13 & 0.28 & 129.7 & 0.09 & 3.753 & -4.10 \\
\hline HD 114911 & $\mathrm{~B}$ & 4.84 & 4.14 & 3.96 & 0.88 & 0.22 & 336.6 & -0.49 & 3.690 & -3.14 \\
\hline HD 133880 & B & 3.50 & 3.04 & 2.90 & 1.17 & 0.37 & 154.7 & 0.15 & 3.761 & -4.38 \\
\hline HD $134837^{\mathrm{a}}$ & B & & & 5.76 & 0.18 & 0.06 & 521.7 & -1.55 & 3.508 & -2.68 \\
\hline HD $134946^{\mathrm{a}}$ & $\mathrm{B}$ & & & 7.00 & 0.30 & 0.08 & 1038.0 & -2.18 & 3.432 & -1.62 \\
\hline HD $135734^{\mathrm{a}, \mathrm{c}}$ & $\begin{array}{l}\text { B } \\
\text { C }\end{array}$ & & & $\begin{array}{l}0.57 \\
9.95\end{array}$ & 0.05 & 0.01 & $\begin{array}{c}92.5 \\
547.6\end{array}$ & -3.37 & 3.368 & -0.10 \\
\hline HD $145483^{\mathrm{a}, \mathrm{c}}$ & $\begin{array}{l}\text { B } \\
\text { C }\end{array}$ & & & $\begin{array}{l}2.05 \\
3.14\end{array}$ & 1.08 & 0.37 & $\begin{array}{c}344.3 \\
18.5\end{array}$ & -0.02 & 3.741 & -3.26 \\
\hline HD $159376^{\mathrm{a}}$ & $\begin{array}{l}\mathrm{B} \\
\mathrm{C} \\
\mathrm{D}\end{array}$ & & & $\begin{array}{l}5.50 \\
7.02 \\
7.91 \\
\end{array}$ & $\begin{array}{l}0.54 \\
0.28 \\
0.16 \\
\end{array}$ & $\begin{array}{l}0.13 \\
0.07 \\
0.04 \\
\end{array}$ & $\begin{array}{l}2358.5 \\
1468.8 \\
1718.1 \\
\end{array}$ & $\begin{array}{l}-1.35 \\
-2.01 \\
-2.40\end{array}$ & $\begin{array}{l}3.573 \\
3.531 \\
3.505 \\
\end{array}$ & $\begin{array}{l}-1.74 \\
-1.09 \\
-0.69 \\
\end{array}$ \\
\hline HD $169022^{\mathrm{C}}$ & $\mathrm{B}$ & 3.44 & 3.22 & 3.27 & & & 106.0 & & & \\
\hline HD $169978^{\mathrm{a}}$ & $\mathrm{B}$ & & & 7.86 & 0.15 & 0.03 & 452.9 & -2.40 & 3.504 & -1.20 \\
\hline \multicolumn{11}{|c|}{ Stars already known to be members of visual binary systems } \\
\hline HD $27657^{\mathrm{a}, \mathrm{c}}$ & $\mathrm{B}$ & & & 2.4 & & & 586.6 & & & \\
\hline HD $36408^{a, d}$ & $\mathrm{~B}$ & & & -1.3 & & & 3336.6 & & & \\
\hline HD $78556^{\mathrm{a}, \mathrm{c}}$ & $\mathrm{B}$ & & & 1.76 & & & 358.2 & & & \\
\hline HD $90972^{\mathrm{a}, \mathrm{c}}$ & $\mathrm{B}$ & & & 2.50 & & & 1634.9 & & & \\
\hline HD $119055^{\mathrm{a}}$ & $\mathrm{B}$ & & & 3.37 & 1.02 & 0.40 & 435.0 & -0.12 & 3.730 & -3.53 \\
\hline HD $184707^{\mathrm{a}}$ & $\mathrm{B}$ & & & 3.77 & 0.92 & 0.33 & 141.2 & -0.36 & 3.700 & -3.01 \\
\hline
\end{tabular}

a We only have $K$-band photometry. Thus, the absolute $J$ and $H$-magnitudes entries are left open.

b The companion has a very low absolute $K$-magnitude and cannot be fitted by evolutionary models.

c Values for fundamental parameters are presented in Table 6.

d The magnitude difference between the primary and the companion in the $K$-band is only 0.25 mag. Gravitationally bound companion would be a main sequence star with the mass similar to that of primary.

Table 6. Fundamental parameters for the companions on the main sequence.

\begin{tabular}{|c|c|c|c|c|c|c|c|}
\hline \multicolumn{10}{|c|}{ Detected visual systems } \\
\hline \hline HD & Comp. & $M / M_{\odot}$ & $q$ & $\log \left(L / L_{\odot}\right)$ & $\log \left(T_{\text {eff }}\right)$ & $\log \left(L_{\mathrm{X}} / L_{\text {bol }}\right)$ & Stellar model used \\
\hline \hline HD 32964 & B & 0.67 & 0.23 & -0.94 & 3.640 & $\leq-2.88$ & Charbonnel et al. (1999) \\
\hline HD 33802 & B & $\geq 1.05$ & $\leq 0.29$ & $\geq-0.06$ & $\geq 3.763$ & $\leq-2.85$ & Schaller et al. (1992) \\
\hline HD 135734 & B & $>1.00$ & $<0.26$ & $>-1.16$ & $>3.751$ & $<-3.31$ & Schaller et al. (1992) \\
\hline HD 145483 & B & $\geq 1.28$ & $\leq 0.44$ & $\geq 0.37$ & $\geq 3.812$ & $\leq-3.66$ & Schaller et al. (1992) \\
\hline HD 169022 & B & 0.95 & 0.27 & -0.05 & 3.764 & -4.20 & Schaller et al. (1992) \\
\hline \hline \multicolumn{8}{|c|}{ Stars already known to be members of visual binary systems } \\
\hline HD 27657 & B & $\geq 1.18$ & $\geq 0.35$ & $\geq 0.20$ & $\geq 3.792$ & $\leq-4.20$ & Schaller et al. (1992) \\
\hline HD 78556 & B & $\geq 1.40$ & $\geq 0.36$ & $\geq 0.55$ & $\geq 3.835$ & $\leq-3.84$ & Schaller et al. (1992) \\
\hline HD 90972 & B & $\geq 1.15$ & $\geq 0.34$ & $\geq 0.14$ & $\geq 3.785$ & $\leq-3.86$ & Schaller et al. (1992) \\
\hline
\end{tabular}




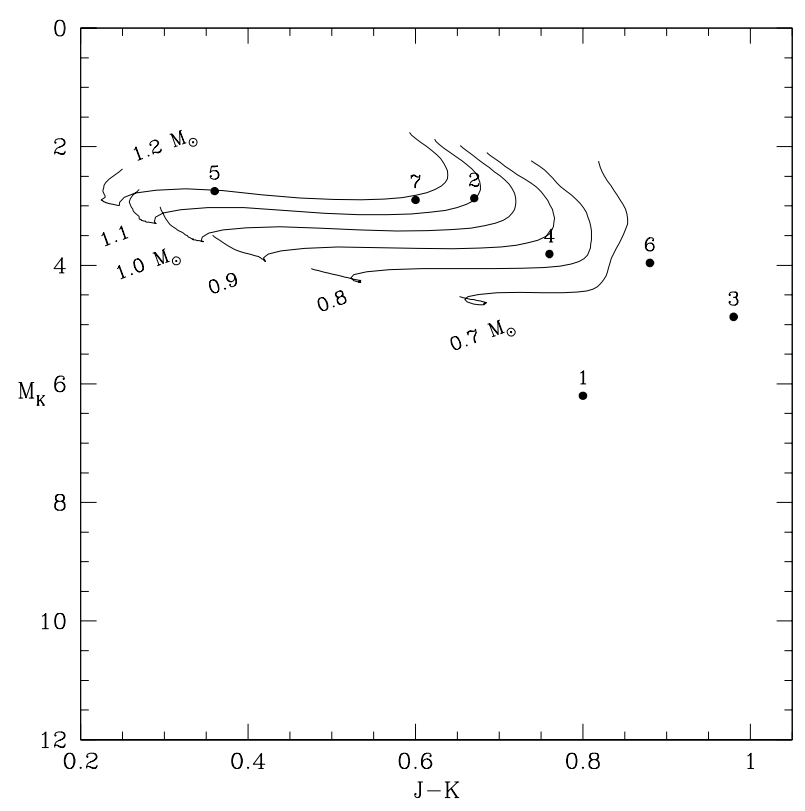

Fig. 3. Position of companions in the $M_{K}-\left(M_{J}-M_{K}\right)$ colormagnitude diagram for PMS stars using the set B98 $8_{\text {Sun }}$.

In Cols. 2-4 of Table 5 we present absolute $J, H$ and $K$ magnitudes for the companions. The masses of PMS companions and corresponding luminosities and effective temperatures were obtained by interpolation in the evolutionary tracks of the models B98 LMS. As our systems are located at rather short distances, they are unlikely to exhibit significant interstellar reddening. Nevertheless, we have computed the colour excess $E[B-V]$ of the B stars in the Geneva system using the calibration of Cramer (1982) and obtained the more widely used $E(B-V)$ colour excess of Johnson's system through the relation $E(B-V)=0.842 E[B-V]$ (Cramer 1984). One object not measured in the Geneva system but having uvby $\beta$ colours in the literature (HD 119055) has $E(b-y)=0.028$ according to Crawford's (1978) calibration, which implies $E(B-V)=0.036$. In this way we could verify that all but 4 stars have $E(B-V) \leq 0.05$, the others having $E(B-V) \sim 0.08$ to 0.17 . The largest colour excess is that of HD 159376, a Si star, so it is not very reliable because of the anomalous colours. In this worst case, the extinctions in the $J, H$ and $K$ bands are respectively $0.15,0.09$ and 0.06 mag. Therefore null extinction was assumed in calculation of absolute $J H K$ magnitudes. In Col. 6 we give the companion masses interpolated from theoretical evolutionary models calculated by Baraffe et al. (1998). The mass-ratios in the systems are presented in Col. 7. From the B98 models inferred luminosities and effective temperatures are given in Cols. 8 and 9 . The last column displays the expected ratios $\log \left(L_{\mathrm{X}} / L_{\mathrm{Bol}}\right)$.

In Table 6 we present fundamental parameters of the companions on the main sequence. For companions with masses $\geq 0.8 M_{\odot}$ we used evolutionary tracks of Schaller et al. (1992), which cover the range of 0.8 to $120 M_{\odot}$, to estimate $\log \left(L / L_{\odot}\right), \log \left(T_{\text {eff }}\right)$ and $\log \left(L_{\mathrm{X}} / L_{\text {bol }}\right)$. For companions with lower masses we used the evolutionary tracks of Charbonnel et al. (1999). As in the paper of Berghöfer et al. (1996) we adopt for calculation of $\log \left(L_{\mathrm{X}} / L_{\mathrm{bol}}\right)$ a typical error of 0.3 dex.

\subsection{Notes on stars}

In the following we give a brief overview of the studied systems.

$H D 1685=H R$ 83: We cannot fit the companion with the B98 models. It is quite possible that this system does not form a physical pair. Using the magnitude $\mathrm{M}_{K}$ alone and assuming the coeval formation of the system we obtain for the mass of the companion the value $0.6 M_{\odot}$. We calculate a fractional X-ray luminosity of $\log \left(L_{\mathrm{X}} / L_{\mathrm{Bol}}\right)=-2.60$ which is above the saturation limit.

$H D 21790=H R$ 1070: This system is the widest system in our sample. No images with the $J$ and $H$ filters were taken and the probability to find a background star of such a faint magnitude of 16.2 is high. If we assumed that this system is a physical pair, the mass of the companion inferred from the absolute $K$-magnitude would be very low $\left(M=0.04 M_{\odot}\right)$ and the companion would be expected to be a brown dwarf. Another problem is that in either case (background object or physical pair) the companion discovered here cannnot produce the observed $\mathrm{X}$-ray luminosity. We suggest that the X-radiation very likely originates from an additional close unresolved latetype companion with coronal X-ray emission.

$H D 27376=H R$ 134\%: This star is an SB2 star with an orbital period of 5.0 days and a mass ratio close to 1.0 (Batten et al. 1989). The estimated angular separation of the components of this system is $0 . ' 0016$ (Halbwachs 1981). According to the B98 $8_{\text {LMS }}$ model the detected companion is a PMS star. Two additional companions at $49^{\prime \prime} .4$ and at $00^{\prime \prime} 2$ are mentioned in the Hipparcos Input Catalogue (CDS Catalogue I/196), (henceforth HIC) and in the BSC. Thus, this system could consist of five stars. We calculate a fractional X-ray luminosity of $\log \left(L_{\mathrm{X}} / L_{\mathrm{Bol}}\right)=-3.42$. This value is well below the saturation limit of $\log \left(L_{\mathrm{X}} / L_{\mathrm{Bol}}\right) \approx-3$.

$H D 29589=H R$ 1484: The companion has a very low $M_{K}$ magnitude (12.2) and cannot be fitted by B98 models. No images with the $J$ and $H$ filters were taken and the probability to find a background star of such a faint magnitude is very high.

$H D$ 32964 = HR 165\%: This star is an SB2 star with an orbital period of 5.5 days and a mass ratio close to 1.0 (Batten et al. 1989). The estimated angular separation of the component of this system is $0 . \prime 0012$ (Halbwachs 1981). We cannot fit the detected companion with the model B98 ${ }_{\text {LMS }}$. Therefore, we assume that it is rather a main sequence star with a mass of $0.67 M_{\odot}$ computed from the MLR relation of Henry \& McCarthy (1993), even though it might be a $\sim 0.7 M_{\odot}$ PMS star according to the B98 Sun model. The companion's fractional X-ray luminosity amounts to $\log \left(L_{\mathrm{X}} / L_{\mathrm{Bol}}\right)=-2.88$, a value which is close to the saturation limit of $\log \left(L_{\mathrm{X}} / L_{\mathrm{Bol}}\right) \approx-3$. An 
additional companion at the angular distance of $52^{\prime \prime} 2$ is mentioned in the HIC and in the BSC. Hence, this system may be, in fact, a quadruple system.

$H D$ 33802 = HR 1696: The companion is not a PMS star. Using the $M_{(J H K)}$-mass relations given by Henry \& McCarthy (1993) for main-sequence stars we estimate the mass of the companion to be $\geq 1.05 M_{\odot}$. As these relations were determined only for stars with masses 0.08 to $1.0 M_{\odot}$, only a lower limit of the mass of the companion can be estimated. As far as we know, the infrared MLRs have not been extended to higher masses through other studies. According to the Sch92 models, the detected companion could be a very young main-sequence star with a fractional X-ray luminosity of $\log \left(L_{\mathrm{X}} / L_{\mathrm{Bol}}\right) \leq-2.85$. An additional companion at $122^{\prime \prime} 1$ is mentioned in the HIC and in the BSC. Therefore, this system could be a triple system.

$H D 73340=H R$ 3413: According to the B98 $8_{\text {LMS }}$ models the companion is a PMS star with a mass of $1.20 M_{\odot}$. This system belongs to the open cluster IC 2391 (BSC).

$H D$ 73952 = HR 3435: We cannot fit the companion with the B98 models. It is quite possible that this system does not form a physical pair. Using the magnitude $M_{K}$ alone and assuming coeval formation of the system we obtain for the mass of the companion the value $0.62 M_{\odot}$. We calculate a fractional X-ray luminosity of $\log \left(L_{\mathrm{X}} / L_{\mathrm{Bol}}\right)=-3.02$ which is very close to the saturation limit. The star HD 73952 belongs to the open cluster IC 2391 (BSC). An additional companion at $22^{\prime \prime} .3$ is mentioned in the HIC and in the BSC.

$H D 75333=H R$ 3500: According to the B98 $8_{\mathrm{LMS}}$ models the companion is a PMS star. We calculate a fractional X-ray luminosity of $\log \left(L_{\mathrm{X}} / L_{\mathrm{Bol}}\right)=-3.00$, which is at the level of the saturation limit. This system possibly belongs to the Pleiades Group (BSC).

$H D 110073=H R$ 481\%: This star is an SB1 star (Schneider 1981). According to the B98 $8_{\mathrm{LMS}}$ models the companion is a PMS star. This system belongs to the Pleiades group (BSC).

$H D 114911=H R$ 4993: This is an SB2 star with an orbital period of 20.0 days and a mass ratio close to one (Batten et al. 1989). The mass of the companion obtained by interpolation in B98 $8_{\text {LMS }}$ models is $0.57 M_{\odot}$. However the age of the companion with such a mass based on isochrone fitting $\left(0.01 \times 10^{8}\right)$ is in strong disagreement with the age of the primary star $\left(1.31 \times 10^{8}\right)$. Using the magnitude $M_{K}$ alone and assuming the coeval formation of the system we obtain for the mass of the companion the value $0.88 M_{\odot}$. We calculate a fractional X-ray luminosity of $\log \left(L_{\mathrm{X}} / L_{\mathrm{Bol}}\right)=-3.14$. As an additional companion at $60^{\prime \prime} .2$ is mentioned in the $\mathrm{HIC}$ and in the BSC, this system could be a quadruple system.

$H D 133880=H R$ 5624: According to the B98 ${ }_{\mathrm{LMS}}$ models the companion is a PMS star with a mass of $1.17 M_{\odot}$. This system belongs to the Scorpius-Centaurus OB association (BSC).

$H D 134837=H R$ 5653: No images were taken for this star with the $J$ and $H$ filters. If we assume that the companion is gravitationally bound and not a background object, we obtain from B98 of the companions is $0.18 M_{\odot}$. We calculate a fractional X-ray luminosity of $\log \left(L_{\mathrm{X}} / L_{\mathrm{Bol}}\right)=-2.68$ which is above the saturation level.

$H D 134946=H R$ 5655: No images were taken for this star with the $J$ and $H$ filters. If we assume a coeval formation of this system we obtain, according to the B98 $8_{\text {LMS }}$ models, that the companion is a PMS star with a mass of $0.30 M_{\odot}$. We have derived a fractional X-ray luminosity of $\log \left(L_{\mathrm{X}} / L_{\mathrm{Bol}}\right)=-1.62$. This value is much larger than the saturation limit of $\log \left(L_{\mathrm{X}} / L_{\mathrm{Bol}}\right) \approx-3$. It is possible that the $\mathrm{X}$ radiation might originate from an additional close, unresolved low-mass companion with coronal X-ray emission.

$H D 135734=H R$ 5683: Only $K$ imaging has been done for this system. Under the assumption of a coeval formation we obtain, according to the B98 $8_{\text {LMS }}$ models, that the companion at the angular distance of $66^{\prime \prime} 15$ is a PMS star of the mass $0.05 M_{\odot}$. Given the very low mass inferred from the absolute $K$-magnitude, the faint companion could be a brown dwarf. We have derived a fractional X-ray luminosity of $\log \left(L_{\mathrm{X}} / L_{\mathrm{Bol}}\right)=-0.16$. This value is much larger than the saturation $\operatorname{limit}$ of $\log \left(L_{\mathrm{X}} / L_{\mathrm{Bol}}\right) \approx-3$. This system is likely a quadruple system. The companion observed at 1".0 was already known (HIC \& BSC) and, according to B98 models, it is not a PMS star. We estimate for this companion a mass $>1.00$ and calculate a fractional X-ray luminosity of $\log \left(L_{\mathrm{X}} / L_{\mathrm{Bol}}\right)<-3.31$. An additional companion at $23 \prime .5$ is mentioned in the HIC and in the BSC. We suggest that X-radiation very likely originates either from the already known companion at 1."0 or from the companion at the angular distance of $233^{\prime \prime} 5$. This system belongs to the Scorpius-Centaurus OB association (BSC).

HD 145483=HR 6029: No images were taken with the $J$ and $H$ filters. The companion at the angular distance of 3 3. $^{\prime}$ was already known (HIC \& BSC). According to the B98 LMS models it is not a PMS star. Using $M_{(J H K)}$-mass relations given by Henry \& McCarthy (1993) for mainsequence stars, we estimate that the mass of the companion is $\geq 1.28$. According to the Sch92 models the known companion is a ZAMS star. The companion discovered at the angular distance of $0^{\prime \prime} .2$ has a mass $M=1.08 M_{\odot}$ and is a PMS star according to the B98 $8_{\text {LMS }}$ models. This triple system belongs to the upper Scorpius region (BSC).

$H D 159376=H R$ 6545: Only $K$ imaging has been done for this system. The field around this star is very crowded and the probability of finding a background star is high. If we assume the coeval formation of this system, we obtain, according to the B98 $8_{\text {LMS }}$ models, that all three companions are PMS stars. The calculated fractional $\mathrm{X}$-ray luminosity of all three companions is much larger than the saturation limit of $\log \left(L_{\mathrm{X}} / L_{\mathrm{Bol}}\right) \approx-3$. In other words, the detected companions alone cannot produce the observed X-ray luminosity.

HD 169022 = HR 6879: According to the B98 models the companion is not a PMS star. From the empirical mass estimate using the $M_{(J H K)}$-mass relations given by Henry \& McCarthy (1993) we obtain the value $0.95 M_{\odot}$. Using 
Sch92 models we find that the companion is a ZAMS star. An additional companion at the angular distance of $32 . \prime 3$ is mentioned in $\mathrm{HIC}$ and BSC.

$H D 169978=H R$ 6916: No images were taken with the $J$ and $H$ filters. According to the B98 ${ }_{\text {LMS }}$ models the companion is a PMS star of $0.15 M_{\odot}$. The calculated fractional X-ray luminosity of the companions is much larger than the saturation limit of $\log \left(L_{\mathrm{X}} / L_{\mathrm{Bol}}\right) \approx-3$.

$H D$ 27657 = HR 1372: No images were taken with the $J$ and $H$ filters. The presence of a companion was already known before we observed this star (HIC \& BSC). According to the B98 models the companion is not a PMS star. From the Henry \& McCarthy's (1993) models we obtain a lower limit for the mass $M \geq 1.18 M_{\odot}$. According to the Sch92 models the companion is a very young main-sequence star.

HD $36408=H R$ 184\%: This is an SB star (BSC). No images were taken with the $J$ and $H$ filters. The visual companion was already known before we observed this star. The magnitude difference $\Delta K$ between the primary and the companion in the $K$-band is very small $(0.25)$. Considering the position of the primary in the $\mathrm{H}-\mathrm{R}$ diagram, we conclude that a gravitationally bound companion should be a main sequence star.

HD $78556=H R$ 3630: This is an SB star (BSC). No images were taken in $J$ and $H$. The visual companion was already known before our observations (HIC \& BSC). According to the B98 models the companion is not a PMS star. Models of Henry \& McCarthy give a lower limit of $M \geq 1.40 M_{\odot}$. According to the Sch92 model the companion could be a ZAMS star.

$H D$ 90972 $=H R$ 4118: This is an SB2 star (BSC) and the companion was known before we observed this system (HIC \&BSC). No images in $J$ and $H$ were taken. According to the B98 models the companion is not a PMS star. For the mass we get $M \geq 1.15 M_{\odot}$. From the Sch92 models, we conclude that the companion could be a ZAMS star.

HD $119055=H R$ 5144: No images were taken with the $J$ and $H$ filters. The companion was already known (HIC \& BSC). According to the B98 ${ }_{\text {LMS }}$ models the companion is a PMS star of the mass $1.02 M_{\odot}$.

$H D 184707=H R$ 7440: Only $K$ imaging has been done for this system. The companion was already known (HIC \& BSC). According to the B98 $8_{\mathrm{LMS}}$ models the companion is a PMS star of the mass $0.92 M_{\odot}$.

\section{Discussion}

The results of our study clearly confirm our suspicion that most late B-type stars which are detected in X-rays are accompanied by a low-mass late-type companion. Although we have no formal evidence that the X-ray emission is due the low-mass companion (e.g. spectra showing that the companions are indeed active, or lack of active lowmass companions in a control sample of B stars which are not X-ray sources), this hypothesis appears strongly backed by our data. If we admit that the late type companions are gravitationally bound in the studied systems, we expect that out of 19 detected companions, 15 are PMS stars. Among the six already known visual binary systems, two contain a PMS companion. A very interesting project would be to investigate these systems more closely with near infrared spectrographs used together with adaptive optics systems. Such spectroscopic studies would provide a much more accurate estimate of the stellar masses of the components through their spectral type and would allow to conclusively determine whether the companion is, indeed, a PMS star or a background star.

The issue whether all studied systems form physical pairs or not deserves further investigation. The detected companions have projected separations ranging from 0.2 to $14^{\prime \prime} 1$ (18-2358 AU). We cannot rule out that the widest systems are not physical ones. Estimates of cut-off separation for the gravitational binding of the pairs by several authors (Chandrasekhar 1944; Bahcall et al. 1986; Duquennoy \& Mayor 1991) place it between $2 \times 10^{3}$ and $2 \times 10^{4}$ AU. The radial velocity measurements and proper motion studies would tell us whether the stars in these systems are gravitationally bound or not. Out of the 49 late B-type stars in our sample, 25 have additional companions in the field of view. Of the studied systems, 6 were already known as visual binaries, and 19 are newly detected by us. The sample of the systems with companions probably consists of 9 binary systems, 5 triple, 4 quadruple systems and one system consisting of 5 stars. That yields an observed binary frequency of $51 \%$ for our star sample.

As mentioned in the Introduction, the formation mechanism of massive stars is not well understood yet, and it is important to compare the multiplicity of higher mass stars to that of lower mass stars. However, massive stars are less frequent than low mass stars and the large brightness of the massive primaries prevents the detection of close visual low mass companions. Previous studies of the spectroscopic binary frequency of B stars have shown that on average the percentage of spectroscopic binaries is higher than among solar type stars (e.g., Abt et al. 1990; Morell \& Levato 1991). McAlister et al. (1993) carried out speckle interferometric observations for 2088 OB stars and found a binary frequency of B stars of about $14 \%$. Bouvier \& Corporon (2001) observed a sample of 63 Herbig Ae/Be stars among which 22 were B0-B8 stars and 34 B9-A9 stars. They found an observed binary frequency for the two groups of stars of, respectively, $36 \%$ and $42 \%$. These values are slightly lower than that in the present work.

In our study, the random pairing, that we estimated to 2 cases over 49 studied stars, might affect the observed binary frequency. On the other hand, very recent results obtained from higher angular resolution imaging on the same star sample using Keck Observatory AO facilities already revealed two new companions (among 8 targets) with a separation of the order of 50 milli-arcseconds (Le Mignant 2001). A very likely reason for the higher binary frequency in the present work is that the sample has been $\mathrm{X}$-ray selected. As a consequence, the multiplicity study in our 
late-B star sample is biased towards low-mass companions (which have strong X-ray emission). For statistical purposes it will be of crucial importance to study in the future a sample of late-B type stars not detected in the ROSAT all-sky survey. Such a study will represent a systematic multiplicity study of early type stars which would set important constraints to any theory of binary and multiple star formation.

Nearly $20 \%$ of the late-B stars found in the ROSAT survey belong to young stellar groups: Sco-Cen association, Sco OB2, and the Pleiades cluster and supercluster. The remaining X-ray emitting B stars are field stars. A further study of X-ray emission of the systems with late Btype primaries, e.g. with the Chandra X-ray Observatory which offers rather high spatial resolution, will provide a good opportunity to examine the link between X-ray emission and age in the stars having different masses and different formation histories.

Acknowledgements. DLM wishes to acknowledge the support from the European Southern Observatory during the data reduction in Garching. We would like to thank Dr. Isabelle Baraffe (École Polytechnique of Lyon) for providing her stellar evolutionary models and Dr. Fiorella Castelli for calculation of effective temperatures of a few late B-type stars using Strömgren photometry. Observing support during direct and coronagraphic imaging observations from the $3.6 \mathrm{~m}$ telescope team is also gratefully acknowledged, especially Victor Merino, Dr. Franck Marchis and Dr. Olivier Marco (ESO La Silla/Santiago). PN acknowledges the support of the Swiss National Science Foundation. Finally, we wish to thank Patricia Goudé from Keck Observatory for the technical editing work she did on the paper, and the referee, Dr. F. Walter, for constructive comments.

\section{References}

Abt, H. A., Gomez, A. E., \& Levy, S. G. 1990, ApJS, 74, 551 Bahcall, J. N., Ratnatunga, K. U., \& Jones, B. F. 1986, ApJS, 60, 939

Baraffe, I., Chabrier, G., Allard, F., \& Hauschildt, P. H. 1998, A\&A, 337, 403

Batten, A. H., Fletcher, J. M., \& MacCarthy, D. G. 1989, Eighth Catalogue of the Orbital Elements of Spectroscopic Binary Systems, Publ. Dominion Astrophys. Obs., 17

Berghöfer, T. W., \& Schmitt, J. H. M. M. 1994, A\&A, 292, L5

Berghöfer, T. W., Schmitt, J. H. M. M., \& Cassinelli, J. P. 1996, A\&AS, 118, 481

Berghöfer, T. W., Schmitt, J. H. M. M., Danner, R., \& Cassinelli, J. P. 1997, A\&A, 322, 167

Berghöfer, T. W., \& Schmitt, J. H. M. M. 2001, in preparation

Bouvier, J., \& Corporon, P. 2001, in The Formation of Binary Stars, Proceedings of the 200th Symposium of the International Astronomical Union, ed. H. Zinnecker, \& R. D. Mathieu, in press

Brandner, W., Alcala, J., Kunkel, M., Moneti, A., \& Zinnecker, H. 1996, A\&A, 307, 121

Brandner, W., Alcala, J. M., Covino, E., \& Frink, S. 1997, AAS, 190, 41.04

Burki, G., et al. 2001, GENEVA photometric database, http://obswww. unige.ch/gcpd/ph13.html
Burnet, M., \& Rufener, F. 1979, A\&A, 74, 54

Castelli, F. 2000, in preparation

Chandrasekhar, S. 1944, ApJ, 99, 54

Charbonnel, C., Däppen, W., Schaerer, D., et al. 1999, A\&AS, 135,405

Cramer, N. 1982, A\&A, 112, 330

Cramer, N. 1984, A\&A, 132, 283

Cramer, N. 1999, New Astron. Rev., 43, 343

Cramer, N., \& Maeder, A. 1979, A\&A, 78, 305

Crawford, D. L. 1978, AJ, 83, 48

D'Antona, F., \& Mazzitelli, I. 1994, ApJS, 90, 467

Devillard, N. 1997, The Messenger, 87, 19

Duquennoy, A., \& Mayor, M. 1991, A\&A, 248, 485

Eggen, O. J. 1995, AJ, 110, 1749

Epchtein, N. 1998, in New Horizons from Multi-Wavelength Sky Surveys, Proceedings of the 179th Symposium of the International Astronomical Union, ed. B. J. McLean, D. Golombek, J. J. E. Hayes, \& H. E. Payne, 106

FitzGerald, M. P. 1970, A\&A, 4, 234

Gahm, G. F., Ahlin, P., \& Lindroos, K. P. 1983, A\&AS, 51, 143

Ghez, A. M., Neugebauer, G., \& Matthews, K. 1993, PASP, 105,951

Golay, M. 1980, Vistas Astron., 24, 141

Grillo, F., Sciortino, S., Micela, G., Vaiana, G. S., \& Harnden, F. R. 1992, ApJS, 81, 795

Halbwachs, J.-L. 1981, A\&AS, 44, 47

Henry, T. J., \& McCarthy, D. W., Jr. 1993, AJ, 106, 773

Hoffleit, D., \& Jaschek, C. 1982, The Bright Star Catalogue, Yale University Observatory, New Haven, Connecticut, USA

Huelamo, N., Neuhäuser, R., Stelzer, B., Supper, R., \& Zinnecker, H. 2000, A\&A, 359, 227

Köhler, R., \& Leinert, C. 1998, A\&A, 331, 977

Künzli, M., North, P., Kurucz, R. L., \& Nicolet, B. 1997, A\&A, 122,51

Lada, Ch. J., \& Adams, F. C. 1992, ApJ, 393, 278

Le Mignant, D. 2001, unpublished

Leinert, Ch., Zinnecker, H., Weitzel, N., et al. 1993, A\&A, 278, 129

Lindroos, K. P. 1986, A\&A, 156, 223

Mazeh, T., Goldberg, D., Duquennoy, A., \& Mayor, M. 1992, ApJ, 401, 265

Mazzitelli, I. 1992, private communication

McAlister, H. A., Mason, B. D., Hartkopf, W. I., \& Shara, M. M. 1993, AJ, 106, 1639

Mermilliod, J. C., Mermilliod, M., \& Hauck, B. 1997, A\&AS, 124,349

Moon, T. T. 1985, Commun. Univ. London Obs., 78

Morrell, N., \& Levato, H. 1991, ApJS, 75, 965

Neuhäuser, R., Sterzik, M. F., Schmitt, J. H. M. M., Wichmann, R., \& Krautter, J. 1995, A\&A, 297, 391

North, P. 1998, in Highlights of Astronomy, ed. J. Andersen, vol. $11 \mathrm{~B}, 657$

Oppenheimer, B. R., Basri, G., Nakajima, T., \& Kulkarni, S. R. 1997, AJ, 113, 296

Pallavicini, R. 1989, A\&AR, 1, 177

Randich, S., Schmitt, J. H. M. M., Prosser, C. F., et al. 1995, A\&A, 300, 134

Rufener, F. 1988, GENEVA Photometric Catalogue, 4th edition, Observatoire de Genève

Rufener, F., \& Nicolet, B. 1988, A\&A, 206, 357 
Ruphy, S. 1998, in New Horizons from Multi-Wavelength Sky Surveys, Proceedings of the 179th Symposium of the International Astronomical Union, ed. B. J. McLean, D. Golombek, J. J. E. Hayes, \& H. E. Payne, 231

Ruphy, S., Epchtein, N., Cohen, M., et al. 1997, A\&A, 326, 597

Schaller, G., Schaerer, D., Meynet, G., \& Maeder, A. 1992, A\&AS, 96, 269

Schmidt-Kaler, Th. 1982, Landolt \& Börnstein Catalogue, vol. VI/2b

Schmitt, J. H. M. M. 1997, A\&A, 318, 215
Schneider, H. 1981, A\&AS, 44, 137

Shatskii, N. I., \& Tokovinin, A. A. 1998, Astron. Lett., 24, 673

Shu, F. H., Adams, F. C., \& Lizano, S. 1987, ARA\&A, 25, 23 Skrutskie, M. F. 2000, in The extragalactic infrared background and its cosmological implications, IAU Symp., No. 204, in press

Stauffer, J. R., Caillault, J.-P., Gagne, M., et al. 1994, ApJS, 91, 625

Stauffer, J. R., Hartmann, L. W., Prosser, C. F., et al. 1997, ApJ, 479, 776 